International Journal on Organic Electronics (IJOE) Vol.6, No.3, July 2017

\title{
ON APPRoACH To Optimize MANUFACTURING OF A Transistors WiTH Two SOURCES To DECREASE TheIR Dimensions
}

\author{
E.L. Pankratov \\ Nizhny Novgorod State University, 23 Gagarin avenue, Nizhny Novgorod, 603950, \\ Russia
}

\begin{abstract}
In this paper we introduce an approach to optimize technological process of manufacturing of heterotransistors with two sources to decrease their dimensions. Framework the approach we consider a heterostructure with specific configuration. After manufacturing of the heterostructure we consider doping of several areas of the heterostructure by diffusion or ion implantation. The doping was finished by optimized annealing of dopant and/or radiation defects. We introduce an analytical approach for prognosis of mass transport to obtain the required results.
\end{abstract}

\section{KEYWORDS}

Heterotransistor with two sources; optimization of technological process; analytical approach for prognosis of mass transport.

\section{INTRODUCTION}

One of intensively solving aims of solid state electronics in the present time is increasing of density of elements of integrated circuits due to decreasing of their dimensions and optimization of technological processes. One can also find increasing of speed of functioning of these elements. Dimensions of these elements could be decreased by laser or microwave types annealing of the considered dopants [1-3]. These types of annealing leads to inhomogeneity of diffusion coefficient and another parameters of processes due to inhomogenous distribution of temperature and Arrhenius law. The inhomogeneity of these parameters gives a possibility to decrease dimensions of elements of integrated circuits. Radiation processing [4,5] and/or inhomogeneity of heterostructures [6-9] could be also used to change properties of doped materials.

In this paper we consider manufacturing a heterotransistor with two sources (see Fig. 1). The transistor has been manufactured framework the heterostructure from Figs. 1. The heterostructure include into itself a substrate and an epitaxial layer. Several sections have been manufactured in the epitaxial layer by using other materials so as it is shown on Figs. 1. We consider doping of these sections by diffusion or ion implantation to manufacture the required types of conductivity. After this doping it is required optimized annealing of dopant and/or radiation defects. Framework the paper we analyzed redistribution of dopant and radiation defects for determination of conditions, which correspond to decreasing of dimension of the considered transistor.

\section{Method of Solution}

To solve our aim we analyzed distribution of concentration of dopant in space and time. The distribution has been calculated as solution of the following boundary problem 


$$
\frac{\partial C(x, y, z, t)}{\partial t}=\frac{\partial}{\partial x}\left[D_{C} \frac{\partial C(x, y, z, t)}{\partial x}\right]+\frac{\partial}{\partial y}\left[D_{C} \frac{\partial C(x, y, z, t)}{\partial y}\right]+\frac{\partial}{\partial z}\left[D_{C} \frac{\partial C(x, y, z, t)}{\partial z}\right] .
$$

Boundary and initial conditions for the equations could be written as

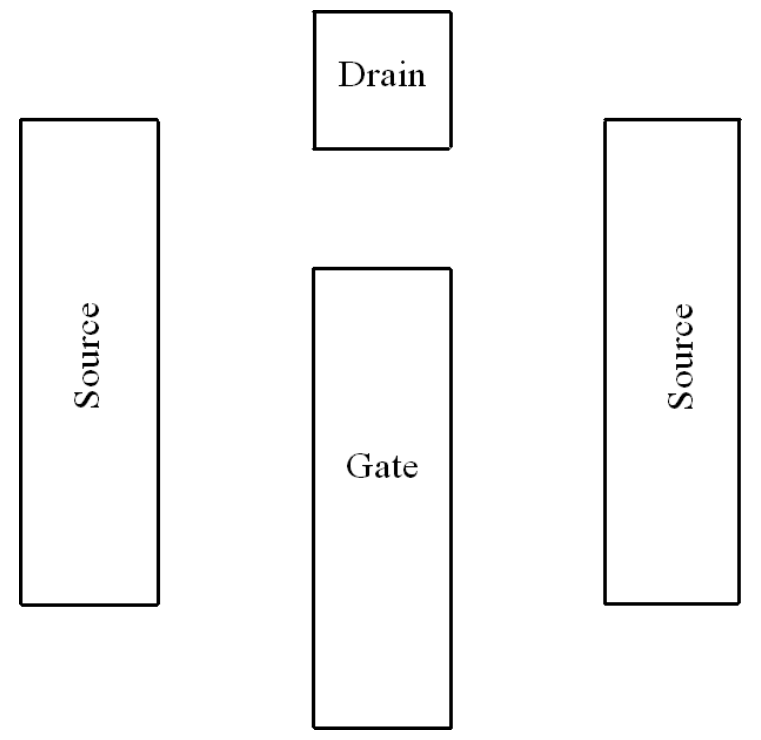

Fig. 1a. Structure of transistor with two sources. View from top

\begin{tabular}{|l|l|l|l|l|l|l|l|l|}
\hline & $n$ & $p$ & $n$ & Epitaxial layer & $n$ & $p$ & $n$ & \\
\hline & Substrate \\
\hline
\end{tabular}

Fig. 1b. Heterostructure with two layers and sections in the epitaxial layer

$$
\begin{aligned}
\left.\frac{\partial C(x, y, z, t)}{\partial x}\right|_{x=0} & =0,\left.\frac{\partial C(x, y, z, t)}{\partial x}\right|_{x=L_{x}}=0,\left.\frac{\partial C(x, y, z, t)}{\partial y}\right|_{y=0}=0,\left.\frac{\partial C(x, y, z, t)}{\partial y}\right|_{x=L_{y}}=0, \\
& \left.\frac{\partial C(x, y, z, t)}{\partial z}\right|_{z=0}=0,\left.\frac{\partial C(x, y, z, t)}{\partial z}\right|_{x=L_{z}}=0, C(x, y, z, 0)=f(x, y, z) .
\end{aligned}
$$

Function $C(x, y, z, t)$ describes the distribution of concentration of dopant in space and time; $T$ is the temperature of annealing; $D_{C}$ is the dopant diffusion coefficient. Value of dopant diffusion coefficient will be changed with changing temperature of annealing. The value will be also changed with changing of concentrations of dopant and radiation defects. All above dependences could be accounted by the following relation $[4,11,12]$

$$
D_{C}=D_{L}(x, y, z, T)\left[1+\xi \frac{C^{\gamma}(x, y, z, t)}{P^{\gamma}(x, y, z, T)}\right]\left[1+\varsigma_{1} \frac{V(x, y, z, t)}{V^{*}}+\varsigma_{2} \frac{V^{2}(x, y, z, t)}{\left(V^{*}\right)^{2}}\right] .
$$


The function $D_{L}(x, y, z, T)$ gives a possibility to take into account dependences of dopant diffusion coefficient on coordinate (due to presents several layers in heterostructure) and temperature (due to Arrhenius law). The function $P(x, y, z, T)$ describes the limit of solubility of dopant. The parameter $1 \leq \gamma \leq 3$ describes quantity of charged defects, which were interacted (in average) with each atom of dopant [11]. The function $V(x, y, z, t)$ describes distribution of concentration of radiation vacancies in space and time with equilibrium distribution $V^{*}$. It is known that diffusion doping of materials did not leads to generation radiation defects. In this situation $\zeta_{1}=\zeta_{2}=0$. We determine spatio-temporal distributions of concentrations of radiation defects by solving the following system of equations $[4,12]$

$$
\begin{aligned}
& \frac{\partial I(x, y, z, t)}{\partial t}=\frac{\partial}{\partial x}\left[D_{I}(x, y, z, T) \frac{\partial I(x, y, z, t)}{\partial x}\right]+\frac{\partial}{\partial y}\left[D_{I}(x, y, z, T) \frac{\partial I(x, y, z, t)}{\partial y}\right]-k_{I, I}(x, y, z, T) \times \\
& \quad \times I^{2}(x, y, z, t)+\frac{\partial}{\partial z}\left[D_{I}(x, y, z, T) \frac{\partial I(x, y, z, t)}{\partial z}\right]-k_{I, V}(x, y, z, T) I(x, y, z, t) V(x, y, z, t) \quad \text { (4) } \\
& \frac{\partial V(x, y, z, t)}{\partial t}=\frac{\partial}{\partial x}\left[D_{V}(x, y, z, T) \frac{\partial V(x, y, z, t)}{\partial x}\right]+\frac{\partial}{\partial y}\left[D_{V}(x, y, z, T) \frac{\partial V(x, y, z, t)}{\partial y}\right]-k_{V, V}(x, y, z, T) \times \\
& \quad \times V^{2}(x, y, z, t)+\frac{\partial}{\partial z}\left[D_{V}(x, y, z, T) \frac{\partial V(x, y, z, t)}{\partial z}\right]-k_{I, V}(x, y, z, T) I(x, y, z, t) V(x, y, z, t) .
\end{aligned}
$$

Boundary and initial conditions for these equations are

$$
\begin{gathered}
\left.\frac{\partial \rho(x, y, z, t)}{\partial x}\right|_{x=0}=0,\left.\frac{\partial \rho(x, y, z, t)}{\partial x}\right|_{x=L_{x}}=0,\left.\frac{\partial \rho(x, y, z, t)}{\partial y}\right|_{y=0}=0,\left.\frac{\partial \rho(x, y, z, t)}{\partial y}\right|_{y=L_{y}}=0, \\
\left.\frac{\partial \rho(x, y, z, t)}{\partial z}\right|_{z=0}=0,\left.\frac{\partial \rho(x, y, z, t)}{\partial z}\right|_{z=L_{z}}=0, \rho(x, y, z, 0)=f_{\rho}(x, y, z) .
\end{gathered}
$$

Here $\rho=I, V$. The function $I(x, y, z, t)$ describes variation of distribution of concentration of radiation interstitials in space and time. The function $D_{\rho}(x, y, z, T)$ describes spatial and temperature dependences of point radiation defects diffusion coefficients. Terms $V^{2}(x, y, z, t)$ and $I^{2}(x, y, z, t)$ describes generation simplest complexes of radiation defects (i.e. divacancies and diinterstitials); the function $k_{I, V}(x, y, z, T)$ describes special and temperature dependences of the parameter of recombination of point radiation defects; the function $k_{I, I}(x, y, z, T)$ and $k_{V, V}(x, y, z, T)$ describe special and temperature dependences of parameters of generation of simplest complexes of point radiation defects.

We determine concentrations of divacancies $\Phi_{V}(x, y, z, t)$ and dinterstitials $\Phi_{I}(x, y, z, t)$ as functions of space and time by solving the following system of equations $[4,12]$

$$
\begin{aligned}
& \frac{\partial \Phi_{I}(x, y, z, t)}{\partial t}=\frac{\partial}{\partial x}\left[D_{\Phi I}(x, y, z, T) \frac{\partial \Phi_{I}(x, y, z, t)}{\partial x}\right]+\frac{\partial}{\partial y}\left[D_{\Phi I}(x, y, z, T) \frac{\partial \Phi_{I}(x, y, z, t)}{\partial y}\right]+ \\
& +\frac{\partial}{\partial z}\left[D_{\Phi I}(x, y, z, T) \frac{\partial \Phi_{I}(x, y, z, t)}{\partial z}\right]+k_{I, I}(x, y, z, T) I^{2}(x, y, z, t)-k_{I}(x, y, z, T) I(x, y, z, t) \\
& \frac{\partial \Phi_{V}(x, y, z, t)}{\partial t}=\frac{\partial}{\partial x}\left[D_{\Phi V}(x, y, z, T) \frac{\partial \Phi_{V}(x, y, z, t)}{\partial x}\right]+\frac{\partial}{\partial y}\left[D_{\Phi V}(x, y, z, T) \frac{\partial \Phi_{V}(x, y, z, t)}{\partial y}\right]+ \\
& +\frac{\partial}{\partial z}\left[D_{\Phi V}(x, y, z, T) \frac{\partial \Phi_{V}(x, y, z, t)}{\partial z}\right]+k_{V, V}(x, y, z, T) V^{2}(x, y, z, t)-k_{V}(x, y, z, T) V(x, y, z, t) .
\end{aligned}
$$


Boundary and initial conditions for these equations are

$$
\begin{gathered}
\left.\frac{\partial \Phi_{\rho}(x, y, z, t)}{\partial x}\right|_{x=0}=0,\left.\frac{\partial \Phi_{\rho}(x, y, z, t)}{\partial x}\right|_{x=L_{x}}=0,\left.\frac{\partial \Phi_{\rho}(x, y, z, t)}{\partial y}\right|_{y=0}=0,\left.\frac{\partial \Phi_{\rho}(x, y, z, t)}{\partial y}\right|_{y=L_{y}}=0, \\
\left.\frac{\partial \Phi_{\rho}(x, y, z, t)}{\partial z}\right|_{z=0}=0,\left.\frac{\partial \Phi_{\rho}(x, y, z, t)}{\partial z}\right|_{z=L_{z}}=0, \Phi_{I}(x, y, z, 0)=f_{\Phi I}(x, y, z), \Phi_{V}(x, y, z, 0)=f_{\Phi V}(x, y, z) .(7)
\end{gathered}
$$

Here functions $D_{\Phi \rho}(x, y, z, T)$ describe spatial and temperature dependences of the diffusion coefficients of the considered complexes of radiation defects; functions $k_{I}(x, y, z, T)$ and $k_{V}(x, y, z, T)$ describe spatial and temperature dependences of the parameters of decay of these complexes. To solve equations for concentrations of dopant and radiation defects we used method of averaging of function corrections [13] with decreased quantity of iteration steps [14]. All calculation procedure is presented in the Appendix.

\section{DISCUSSION}

Now we analyze distribution of concentration of infused (see Fig. $2 a$ ) and implanted (see Fig. $2 b$ ) dopants in space and time in the considered epitaxial layer. Annealing time of dopant for each curve coincides with the same annealing times for another curves framework of each figure. Increasing of number of curve corresponds to increasing of difference between values of dopant diffusion coefficient in layers of heterostructure. Based on these figures one can find that interface between layers of heterostructure gives a possibility to increase absolute value of gradient of concentration of dopant in perpendicular direction to the interface. The variation of the gradient leads to decreasing of dimensions of the considered transistors. At the same time with increasing of absolute value of the above gradient homogeneity of distribution of concentrations of dopants in doped areas increases.

To determine optimal annealing time we take into account variation of the considered gradient of concentration of dopant near interface between epitaxial layer and substrate. With decreasing of value of annealing time one can find increasing of inhomogeneity of distribution of concentration of dopant (see Figs. $3 a$ for diffusion type of doping and $3 b$ for ion type of doping). As optimal annealing time of dopant and/or radiation defects we determine compromise value of the required time time framework recently introduced criterion [15-20]. Based on the criterion we approximate real distribution of concentration of dopant by idealized step-wise function $\psi(x, y, z)$. After the approximation we determine the optimal value of annealing time by minimization of the meansquared error

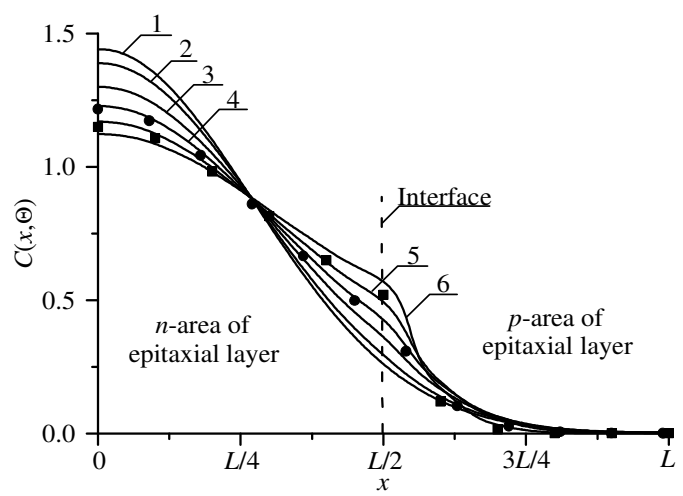

Fig. $2 a$. Dependences of concentration of infused dopant on coordinate in the considered heterostructure in direction, which is perpendicular to interface between epitaxial layer substrate. Difference between values 
of dopant diffusion coefficient in layers of heterostructure increases with increasing of number of curves. Dopant diffusion coefficient in the substrate is smaller than in epitaxial layer. Squares are the experimental data from [21]. Circles are the experimental data from [22]

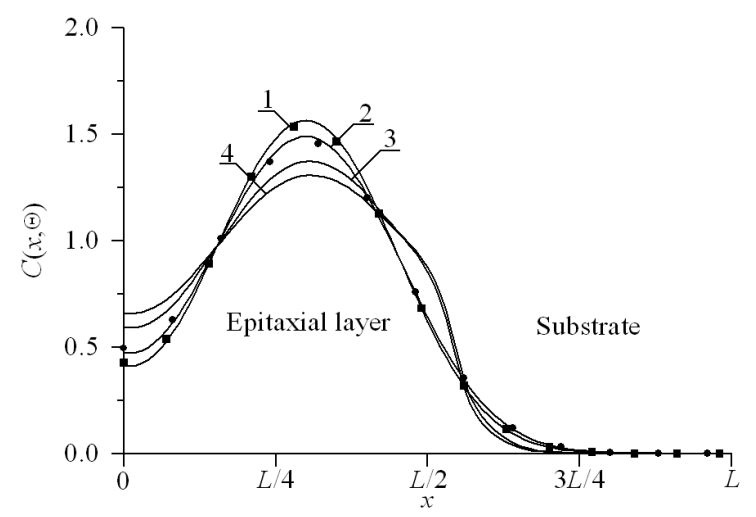

Fig. $2 b$. Dependences of concentration of implanted dopant on coordinate in the considered heterostructure in direction, which is perpendicular to interface between epitaxial layer substrate for two annealing times: $\Theta=0.0048\left(L_{x}{ }^{2}+L_{y}{ }^{2}+L_{z}{ }^{2}\right) / D_{0}$ (for curves 1 and 3) and $\Theta=0.0057\left(L_{x}{ }^{2}+L_{y}{ }^{2}+L_{z}{ }^{2}\right) / D_{0}$ (for curves 2 and 4). Difference between values of dopant diffusion coefficient in layers of heterostructure increases with increasing of number of curves. Dopant diffusion coefficient in the substrate is smaller than in epitaxial layer. Squares are the experimental data from [23]. Circles are the experimental data from [24]

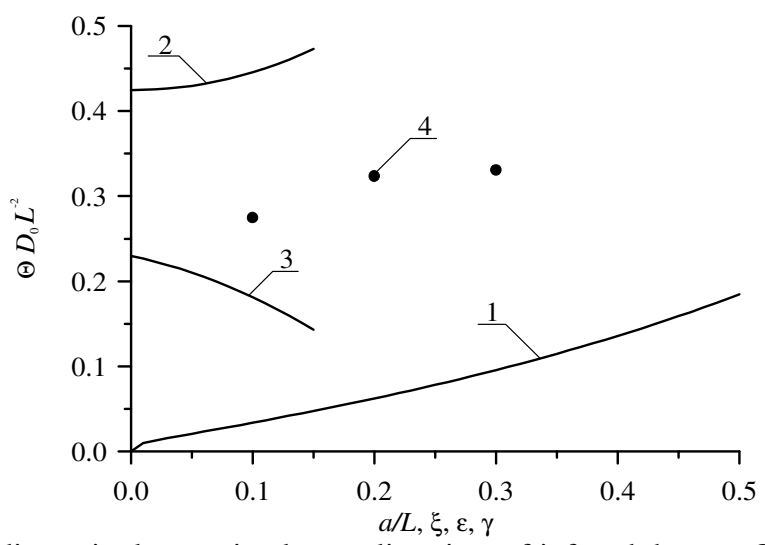

Fig. $3 a$. Dependences of dimensionless optimal annealing time of infused dopant. Curve 1 describes dimensionless optimal annealing time as the function of the relation $a / L$ for $\xi=\gamma=0$ and for equal to each other values of dopant diffusion coefficient in all parts of heterostructure. Curve 2 describes dimensionless optimal annealing time as the function of the parameter $\varepsilon$ for $a / L=1 / 2$ and $\xi=\gamma=0$ and for equal to each other values of dopant diffusion coefficient in all parts of heterostructure. Curve 3 describes dimensionless optimal annealing time as the function of the parameter $\xi$ for $a / L=1 / 2$ and $\varepsilon=\gamma=0$ and for equal to each other values of dopant diffusion coefficient in all parts of heterostructure. Curve 4 describes dimensionless optimal annealing time as the function of the parameter $\gamma$ for $a / L=1 / 2$ and $\varepsilon=\xi=0$ and for equal to each other values of dopant diffusion coefficient in all parts of heterostructure 


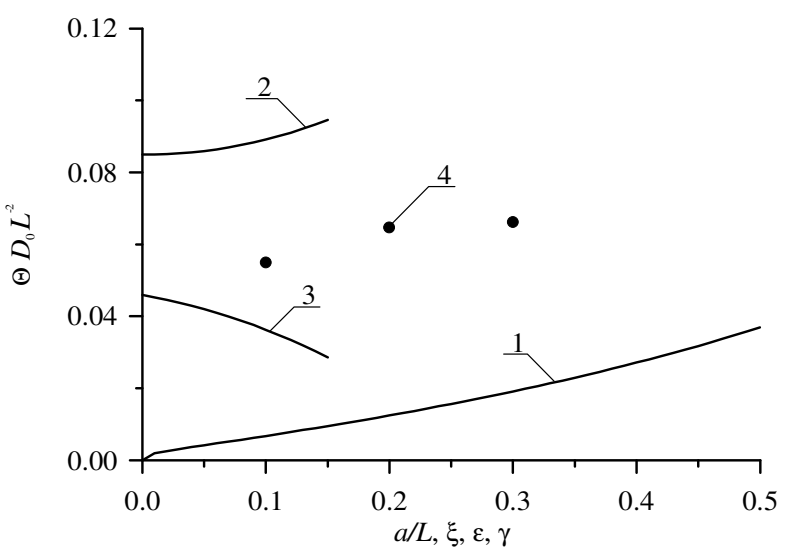

Fig. $3 b$. Dependences of dimensionless optimal annealing time of implanted dopant. Curve 1 describes dimensionless optimal annealing time as the function of the relation $a / L$ for $\xi=\gamma=0$ and for equal to each other values of dopant diffusion coefficient in all parts of heterostructure. Curve 2 describes dimensionless optimal annealing time as the function of the parameter $\varepsilon$ for $a / L=1 / 2$ and $\xi=\gamma=0$ and for equal to each other values of dopant diffusion coefficient in all parts of heterostructure. Curve 3 describes dimensionless optimal annealing time as the function of the parameter $\xi$ for $a / L=1 / 2$ and $\varepsilon=\gamma=0$ and for equal to each other values of dopant diffusion coefficient in all parts of heterostructure. Curve 4 describes dimensionless optimal annealing time as the function of the parameter $\gamma$ for $a / L=1 / 2$ and $\varepsilon=\xi=0$ and for equal to each other values of dopant diffusion coefficient in all parts of heterostructure

$$
U=\frac{1}{L_{x} L_{y} L_{z}} \int_{0}^{L_{x} L_{y} L_{z}} \int_{0}^{0}[C(x, y, z, \Theta)-\psi(x, y, z)] d z d y d x .
$$

Dependences of optimal annealing time are presented on Figs. 3 for both types of doping (diffusion and ion types). It is known, that ion doping of materials leads to necessity to anneal of radiation defects. The annealing leads to spreading of concentration of distribution of dopant during this annealing. In the ideal case distribution of dopant achieves appropriate interfaces between materials of heterostructure during annealing of radiation defects. It should be noted, that it is practicably to additionally anneal the dopant in the case when dopant did not achieves any interfaces during annealing of radiation defects. At the same time ion type of doping gives us possibility to decrease mismatch-induced stress in heterostructure [25].

\section{Conclusions}

We analyzed redistribution of infused and implanted dopants during manufacturing a heterotransistors with two sources. We formulate several recommendations to optimize manufacturing of the heterotransistors to decrease their dimensions. Analytical approach for prognosis of diffusion and ion types of doping with account simultaneous changing of parameters in space and time has been introduced. At the same time nonlinearity of the doping processes could be taken into account by the approach.

\section{ACKNOWLEDGEMENTS}

This work is supported by the agreement of August 27, 2013 № 02.B.49.21.0003 between The Ministry of education and science of the Russian Federation and Lobachevsky State University of Nizhni Novgorod. 


\section{REFERENCES}

[1] K.K. Ong, K.L. Pey, P.S. Lee, A.T.S. Wee, X.C. Wang, Y.F. Chong. Dopant distribution in the recrystallization transient at the maximum melt depth induced by laser annealing. Appl. Phys. Lett. Vol. 89 (17), 172111-172114 (2006).

[2] H.T. Wang, L.S. Tan, E. F. Chor. Pulsed laser annealing of Be-implanted GaN J. Appl. Phys. Vol. 98 (9), 094901-094905 (2005).

[3] Yu.V. Bykov, A.G. Yeremeev, N.A. Zharova, I.V. Plotnikov, K.I. Rybakov, M.N. Drozdov, Yu.N. Drozdov, V.D. Skupov. Diffusion processes in semiconductor structures during microwave annealing. Radiophysics and Quantum Electronics. Vol. 43 (3). P. 836-843 (2003).

[4] V.V. Kozlivsky. Modification of semiconductors by proton beams (Nauka, Sant-Peterburg, 2003, in Russian).

[5] V.L. Vinetskiy, G.A. Kholodar', Radiative physics of semiconductors. ("Naukova Dumka", Kiev, 1979, in Russian).

[6] G. Volovich. Modern chips UM3Ch class D manufactured by firm MPS. Modern Electronics. Issue 2. P. 10-17 (2006).

[7] A. Kerentsev, V. Lanin. Diffusion processes in semiconductor structures during microwave annealing. Power Electronics. Issue 1. P. 34 (2008).

[8] A.O. Ageev, A.E. Belyaev, N.S. Boltovets, V.N. Ivanov, R.V. Konakova, Ya.Ya. Kudrik, P.M. Litvin, V.V. Milenin, A.V. Sachenko. Au-TiBx-n-6H-SiC Schottky barrier diodes: Specific features of charge transport in rectifying and nonrectifying contacts. Semiconductors. Vol. 43 (7). P. 865-871 (2009).

[9] N.I. Volokobinskaya, I.N. Komarov, T.V. Matyukhina, V.I. Reshetnikov, A.A. Rush, I.V. Falina, A.S. Yastrebov. A study of technological processes in the production of high-power high-voltage bipolar transistors incorporating an array of inclusions in the collector region. Semiconductors. Vol. 35 (8). P. 974-978 (2001).

[10] C. Senthilpari, K. Diwakar, A.K. Singh. Low energy, low latency and high speed array divider circuit using a Shannon theorem based adder cell. Recent Patents on Nanotechnology. Vol. 3 (1). P. 61-72 (2009).

[11] Z.Yu. Gotra. Technology of microelectronic devices (Radio and communication, Moscow, 1991).

[12] P.M. Fahey, P.B. Griffin, J.D. Plummer. Point defects and dopant diffusion in silicon. Rev. Mod. Phys. Vol. 61 (2). P. 289-388 (1989).

[13] Yu.D. Sokolov. About the definition of dynamic forces in the mine lifting. Applied Mechanics. Vol. 1 (1). P. 23-35 (1955).

[14] E.L. Pankratov. Dynamics of delta-dopant redistribution during heterostructure growth The European Physical Journal B. Vol. 57 (3). P. 251-256 (2007).

[15] E.L. Pankratov. Dopant diffusion dynamics and optimal diffusion time as influenced by diffusioncoefficient nonuniformity. Russian Microelectronics. Vol. 36 (1). P. 33-39 (2007).

[16] E.L. Pankratov. Redistribution of dopant during annealing of radiative defects in a multilayer structure by laser scans for production an implanted-junction rectifiers. Int. J. Nanoscience. Vol. 7 (4-5). P. 187-197 (2008).

[17] E.L. Pankratov. Decreasing of depth of implanted-junction rectifier in semiconductor heterostructure by optimized laser annealing. J. Comp. Theor. Nanoscience. Vol. 7 (1). P. 289-295 (2010).

[18] E.L. Pankratov, E.A. Bulaeva. Application of native inhomogeneities to increase compactness of vertical field-effect transistors. J. Comp. Theor. Nanoscience. Vol. 10 (4). P. 888-893 (2013).

[19] E.L. Pankratov, E.A. Bulaeva. Using native inhomogeneity of heterostructure to decrease dimensions of planar field-effect transistors Int. J. Micro-Nano Scale Transp. Vol. 2 (2). P. 117-127 (2011).

[20] E.L. Pankratov, E.A. Bulaeva. Increasing of sharpness of diffusion-junction heterorectifier by using radiation processing. Int. J. Nanoscience. Vol. 11 (5). P. 1250028-1250035 (2012).

[21] Voronina T.I., Lagunova T.S., Kizhaev S.S., Molchanov S.S., Pushnyi B.V., Ya-kovlev Yu.P. (2004). MOCVD growth and Mg-doping of InAs layers. Semiconduc-tors. Vol. 38 (5). P. 537-542.

[22] Masse G., Djessas K. (2003). p-n-junctions in (In,Se)/Cu(In,Ga)(Se,S)2(In,Se)/ Cu(In,Ga)(Se,S)2 photovoltaic systems. J. Appl. Phys. Vol. 94 (10). P. 6985-6987.

[23] Ahlgren T., Likonen J., Slotte J., Räisänen J., Rajatore M., Keinonen J. (1997). Con-centration dependent and independent Si diffusion in ion-implanted GaAs. Phys. Rev. B. Vol. 56 (8). P. 45974603.

[24] Noda T. (2003). Indium segregation to dislocation loops induced by ion implanta-tion damage in silicon. J. Appl. Phys. Vol. 93 (3). P. 1428-1431. 
[25] E.L. Pankratov, E.A. Bulaeva. Decreasing of mechanical stress in a semiconductor heterostructure by radiation processing. J. Comp. Theor. Nanoscience. Vol. 11 (1). P. 91-101 (2014).

\section{APPENDIX}

We solved the considered boundary problems by method of averaging of function corrections [13] with decreased quantity of iteration steps [14]. Framework the approach we consider the initial iterations of these solutions as solutions of linear Eqs. (1), (4) and (6) with averaged values of diffusion coefficients $D_{0 L}, D_{0 I}, D_{0 V}, D_{0 \Phi I}, D_{0 \Phi V}$ :

$$
\begin{gathered}
C_{1}(x, y, z, t)=\frac{F_{0 C}}{L_{x} L_{y} L_{z}}+\frac{2}{L_{x} L_{y} L_{z}} \sum_{n=1}^{\infty} F_{n C} c_{n}(x) c_{n}(y) c_{n}(z) e_{n C}(t), \\
I_{1}(x, y, z, t)=\frac{F_{0 I}}{L_{x} L_{y} L_{z}}+\frac{2}{L_{x} L_{y} L_{z}} \sum_{n=1}^{\infty} F_{n I} c_{n}(x) c_{n}(y) c_{n}(z) e_{n I}(t), \\
V_{1}(x, y, z, t)=\frac{F_{0 C}}{L_{x} L_{y} L_{z}}+\frac{2}{L_{x} L_{y} L_{z}} \sum_{n=1}^{\infty} F_{n C} c_{n}(x) c_{n}(y) c_{n}(z) e_{n V}(t), \\
\Phi_{I 1}(x, y, z, t)=\frac{F_{0 \Phi_{I}}}{L_{x} L_{y} L_{z}}+\frac{2}{L_{x} L_{y} L_{z}} \sum_{n=1}^{\infty} F_{n \Phi_{I}} c_{n}(x) c_{n}(y) c_{n}(z) e_{n \Phi_{I}}(t), \\
\Phi_{V 1}(x, y, z, t)=\frac{F_{0 \Phi_{V}}}{L_{x} L_{y} L_{z}}+\frac{2}{L_{x} L_{y} L_{z}} \sum_{n=1}^{\infty} F_{n \Phi_{V}} c_{n}(x) c_{n}(y) c_{n}(z) e_{n \Phi_{V}}(t),
\end{gathered}
$$

where $e_{n \rho}(t)=\exp \left[-\pi^{2} n^{2} D_{0 \rho} t\left(\frac{1}{L_{x}^{2}}+\frac{1}{L_{y}^{2}}+\frac{1}{L_{z}^{2}}\right)\right], \quad F_{n \rho}=\int_{0}^{L_{x}} c_{n}(u) \int_{0}^{L_{y}} c_{n}(v) \int_{0}^{L_{z}} c_{n}(v) f_{\rho}(u, v, w) d w d v d u, c_{n}(\chi)=$ $\cos \left(\pi n \chi / L_{\chi}\right)$. We consider the above solutions as initial-order approximations of concentrations of dopant and radiation defects.

Approximations of considered concentrations with higher orders (higher than the first-order) could be determine framework standard iterative procedure [13,14]. The procedure based on replacement of the functions $C(x, y, z, t), I(x, y, z, t), V(x, y, z, t), \Phi_{I}(x, y, z, t), \Phi_{V}(x, y, z, t)$ in the right sides of the Eqs. (1), (4) and (6) on the following sums $\alpha_{n}+\rho_{n-1}(x, y, z, t)$. The standard iterative procedure leads to equations for the second-order approximations of concentrations of dopant and radiation defects

$$
\begin{gathered}
\frac{\partial C_{2}(x, y, z, t)}{\partial t}=\frac{\partial}{\partial x}\left(\left[1+\varsigma_{1} \frac{V(x, y, z, t)}{V^{*}}+\varsigma_{2} \frac{V^{2}(x, y, z, t)}{\left(^{*}\right)^{2}}\right]\left\{1+\xi \frac{\left[\alpha_{2 C}+C_{1}(x, y, z, t)\right]^{\gamma}}{P^{\gamma}(x, y, z, T)}\right\} \times\right. \\
\left.\times D_{L}(x, y, z, T) \frac{\partial C_{1}(x, y, z, t)}{\partial x}\right)+\frac{\partial}{\partial y}\left(D_{L}(x, y, z, T)\left[1+\varsigma_{1} \frac{V(x, y, z, t)}{V^{*}}+\varsigma_{2} \frac{V^{2}(x, y, z, t)}{\left(V^{*}\right)^{2}}\right] \times\right. \\
\left.\times\left\{1+\xi \frac{\left[\alpha_{2 C}+C_{1}(x, y, z, t)\right]^{\gamma}}{P^{\gamma}(x, y, z, T)}\right\} \frac{\partial C_{1}(x, y, z, t)}{\partial y}\right)+\frac{\partial}{\partial z}\left(D_{L}(x, y, z, T) \frac{\partial C_{1}(x, y, z, t)}{\partial z} \times\right. \\
\left.\times\left[1+\varsigma_{1} \frac{V(x, y, z, t)}{V^{*}}+\varsigma_{2} \frac{V^{2}(x, y, z, t)}{\left(V^{*}\right)^{2}}\right]\left\{1+\xi \frac{\left[\alpha_{2 C}+C_{1}(x, y, z, t)\right]^{\gamma}}{P^{\gamma}(x, y, z, T)}\right\}\right)
\end{gathered}
$$


International Journal on Organic Electronics (IJOE) Vol.6, No.3, July 2017

$$
\begin{aligned}
& \left\{\begin{array}{l}
\frac{\partial I_{2}(x, y, z, t)}{\partial t}=\frac{\partial}{\partial x}\left[D_{I}(x, y, z, T) \frac{\partial I_{1}(x, y, z, t)}{\partial x}\right]+\frac{\partial}{\partial y}\left[D_{I}(x, y, z, T) \frac{\partial I_{1}(x, y, z, t)}{\partial y}\right]+ \\
+\frac{\partial}{\partial z}\left[D_{I}(x, y, z, T) \frac{\partial I_{1}(x, y, z, t)}{\partial z}\right]-\left[\alpha_{2 I}+I_{1}(x, y, z, t)\right]\left[\alpha_{2 V}+V_{1}(x, y, z, t)\right] \times \\
\times k_{I, V}(x, y, z, T)-k_{I, I}(x, y, z, T)\left[\alpha_{2 I}+I_{1}(x, y, z, t)\right]^{2} \\
\frac{\partial V_{2}(x, y, z, t)}{\partial t}=\frac{\partial}{\partial x}\left[D_{V}(x, y, z, T) \frac{\partial V_{1}(x, y, z, t)}{\partial x}\right]+\frac{\partial}{\partial y}\left[D_{V}(x, y, z, T) \frac{\partial V_{1}(x, y, z, t)}{\partial y}\right]+ \\
+\frac{\partial}{\partial z}\left[D_{V}(x, y, z, T) \frac{\partial V_{1}(x, y, z, t)}{\partial z}\right]-\left[\alpha_{2 I}+I_{1}(x, y, z, t)\right]\left[\alpha_{2 V}+V_{1}(x, y, z, t)\right] \times \\
\times k_{I, V}(x, y, z, T)-k_{V, V}(x, y, z, T)\left[\alpha_{2 V}+V_{1}(x, y, z, t)\right]^{2} \\
\end{array}\right. \\
& \begin{array}{l}
\frac{\partial \Phi_{I 2}(x, y, z, t)}{\partial t}=\frac{\partial}{\partial x}\left[D_{\Phi_{I}}(x, y, z, T) \frac{\partial \Phi_{I 1}(x, y, z, t)}{\partial x}\right]+\frac{\partial}{\partial y}\left[D_{\Phi_{I}}(x, y, z, T) \times\right. \\
\left.\times \frac{\partial \Phi_{I 1}(x, y, z, t)}{\partial y}\right]+\frac{\partial}{\partial z}\left[D_{\Phi_{I}}(x, y, z, T) \frac{\partial \Phi_{I 1}(x, y, z, t)}{\partial z}\right]+k_{I, I}(x, y, z, T) \times \\
\frac{\partial \Phi_{V 2}(x, y, z, t)}{\partial t}=\frac{\partial}{\partial x}\left[D_{\Phi_{V}}(x, y, z, T) \frac{\partial \Phi_{V 1}(x, y, z, t)}{\partial x}\right]+\frac{\partial}{\partial y}\left[D_{\Phi_{V}}(x, y, z, T) \times\right. \\
\left.\times \frac{\partial \Phi_{I 1}(x, y, z, t)}{\partial y}\right]+\frac{\partial}{\partial z}\left[D_{\Phi_{V}}(x, y, z, T) \frac{\partial \Phi_{V 1}(x, y, z, t)}{\partial z}\right]+k_{V, V}(x, y, z, T) \times \\
\times V^{2}(x, y, z, t)-k_{V}(x, y, z, T) V(x, y, z, t)
\end{array}
\end{aligned}
$$

Integration of the left and right sides of Eqs.(8)-(10) gives us possibility to obtain relations for the second-order approximations of concentrations of dopant and radiation defects in final form

$$
\begin{gathered}
C_{2}(x, y, z, t)=\frac{\partial}{\partial x}\left(\int_{0}^{t}\left[1+\varsigma_{1} \frac{V(x, y, z, \tau)}{V^{*}}+\varsigma_{2} \frac{V^{2}(x, y, z, \tau)}{\left(V^{*}\right)^{2}}\right]\left\{1+\xi \frac{\left[\alpha_{2 C}+C_{1}(x, y, z, \tau)\right]^{\gamma}}{P^{\gamma}(x, y, z, T)}\right\} \times\right. \\
\left.\times D_{L}(x, y, z, T) \frac{\partial C_{1}(x, y, z, \tau)}{\partial x}\right) d \tau+\frac{\partial}{\partial y}\left(\int_{0}^{t}\left[1+\varsigma_{1} \frac{V(x, y, z, \tau)}{V^{*}}+\varsigma_{2} \frac{V^{2}(x, y, z, \tau)}{\left(V^{*}\right)^{2}}\right] \times\right. \\
\left.\times D_{L}(x, y, z, T)\left\{1+\xi \frac{\left[\alpha_{2 C}+C_{1}(x, y, z, \tau)\right]^{\gamma}}{P^{\gamma}(x, y, z, T)}\right\} \frac{\partial C_{1}(x, y, z, \tau)}{\partial y} d \tau\right)+f_{C}(x, y, z)+\frac{\partial}{\partial z}\left(\int_{0}^{t} D_{L}(x, y, z, T) \times\right. \\
\left.\times\left[1+\varsigma_{1} \frac{V(x, y, z, \tau)}{V^{*}}+\varsigma_{2} \frac{V^{2}(x, y, z, \tau)}{\left(V^{*}\right)^{2}}\right]\left\{1+\xi \frac{\left[\alpha_{2 C}+C_{1}(x, y, z, \tau)\right]^{\gamma}}{P^{\gamma}(x, y, z, T)}\right\} \frac{\partial C_{1}(x, y, z, \tau)}{\partial z} d \tau\right)(8 a) \\
I_{2}(x, y, z, t)=\frac{\partial}{\partial x}\left[\int_{0}^{t} D_{I}(x, y, z, T) \frac{\partial I_{1}(x, y, z, \tau)}{\partial x} d \tau\right]+\frac{\partial}{\partial y}\left[\int_{0}^{t} D_{I}(x, y, z, T) \frac{\partial I_{1}(x, y, z, \tau)}{\partial y} d \tau\right]+ \\
+\frac{\partial}{\partial z}\left[\int_{0}^{t} D_{I}(x, y, z, T) \frac{\partial I_{1}(x, y, z, \tau)}{\partial z} d \tau\right]-\int_{0}^{t} k_{I, I}(x, y, z, T)\left[\alpha_{2 I}+I_{1}(x, y, z, \tau)\right]^{2} d \tau+ \\
+f_{I}(x, y, z)-\int_{0}^{t} k_{I, V}(x, y, z, T)\left[\alpha_{2 I}+I_{1}(x, y, z, \tau)\right]\left[\alpha_{2 V}+V_{1}(x, y, z, \tau)\right] d \tau \quad(9 a)
\end{gathered}
$$


International Journal on Organic Electronics (IJOE) Vol.6, No.3, July 2017

$$
\begin{gathered}
V_{2}(x, y, z, t)=\frac{\partial}{\partial x}\left[\int_{0}^{t} D_{V}(x, y, z, T) \frac{\partial V_{1}(x, y, z, \tau)}{\partial x} d \tau\right]+\frac{\partial}{\partial y}\left[\int_{0}^{t} D_{V}(x, y, z, T) \frac{\partial V_{1}(x, y, z, \tau)}{\partial y} d \tau\right]+ \\
\left.\times \frac{\partial V_{1}(x, y, z, \tau)}{\partial y} d \tau\right]+\frac{\partial}{\partial z}\left[\int_{0}^{t} D_{V}(x, y, z, T) \frac{\partial V_{1}(x, y, z, \tau)}{\partial z} d \tau\right]-\int_{0}^{t} k_{V, V}(x, y, z, T)\left[\alpha_{2 I}+V_{1}(x, y, z, \tau)\right]^{2} d \tau+ \\
+f_{V}(x, y, z)-\int_{0}^{t} k_{I, V}(x, y, z, T)\left[\alpha_{2 I}+I_{1}(x, y, z, \tau)\right]\left[\alpha_{2 V}+V_{1}(x, y, z, \tau)\right] d \tau \\
\Phi_{I 2}(x, y, z, t)=\frac{\partial}{\partial x}\left[\int_{0}^{t} D_{\Phi_{I}}(x, y, z, T) \frac{\partial \Phi_{I 1}(x, y, z, \tau)}{\partial x} d \tau\right]+\frac{\partial}{\partial y}\left[\int_{0}^{t} D_{\Phi_{I}}(x, y, z, T) \frac{\partial \Phi_{I 1}(x, y, z, \tau)}{\partial y} d \tau\right]+ \\
+\frac{\partial}{\partial z}\left[\int_{0}^{t} D_{\Phi_{I}}(x, y, z, T) \frac{\partial \Phi_{I 1}(x, y, z, \tau)}{\partial z} d \tau\right]+\int_{0}^{t} k_{I, I}(x, y, z, T) I^{2}(x, y, z, \tau) d \tau+ \\
+f_{\Phi_{I}}(x, y, z)-\int_{0}^{t} k_{I}(x, y, z, T) I(x, y, z, \tau) d \tau \\
\Phi_{V 2}(x, y, z, t)=\frac{\partial}{\partial x}\left[\int_{0}^{t} D_{\Phi_{V}}(x, y, z, T) \frac{\partial \Phi_{V 1}(x, y, z, \tau)}{\partial x} d \tau\right]+\frac{\partial}{\partial y}\left[\int_{0}^{t} D_{\Phi_{V}}(x, y, z, T) \frac{\partial \Phi_{V 1}(x, y, z, \tau)}{\partial y} d \tau\right]+ \\
+\frac{\partial}{\partial z}\left[\int_{0}^{t} D_{\Phi_{V}}(x, y, z, T) \frac{\partial \Phi_{V 1}(x, y, z, \tau)}{\partial z} d \tau\right]+\int_{0}^{t} k_{V, V}(x, y, z, T) V^{2}(x, y, z, \tau) d \tau+ \\
+f_{\Phi_{V}}(x, y, z)-\int_{0}^{t} k_{V}(x, y, z, T) V(x, y, z, \tau) d \tau
\end{gathered}
$$

Average values of the considered approximations have been determined by the following relations $[13,14]$

$$
\alpha_{2 \rho}=\frac{1}{\Theta L_{x} L_{y} L_{z}} \int_{0}^{\Theta} \int_{0}^{L_{x} L_{y} L_{z}} \int_{0}^{0}\left[\rho_{0}(x, y, z, t)-\rho_{1}(x, y, z, t)\right] d z d y d x d t
$$

Substitution of approximations $(8 a)-(10 a)$ into the previous relation gives the possibility to obtain relations for the average values $\alpha_{2 \rho}$ in the following final form

$$
\begin{aligned}
& \alpha_{2 C}=\frac{1}{L_{x} L_{y} L_{z}} \int_{0}^{L_{x}} \int_{0}^{L_{y} L_{z}} \int_{0} f_{C}(x, y, z) d z d y d x \\
& \alpha_{2 I}=\frac{1}{2 A_{I I 00}}\left\{\left(1+A_{I V 01}+A_{I I 10}+\alpha_{2 V} A_{I V 00}\right)^{2}-4 A_{I I 00}\left[\alpha_{2 V} A_{I V 10}-A_{I I 20}+A_{I V 11}-\right.\right. \\
& \left.\left.-\frac{1}{L_{x} L_{y} L_{z}} \int_{0}^{L_{L}} \int_{0}^{L_{y} L_{z}} \int_{0} f_{I}(x, y, z) d z d y d x\right]\right\}^{\frac{1}{2}}-\frac{1+A_{I V 01}+A_{I I 10}+\alpha_{2 V} A_{I V 00}}{2 A_{I I 00}} \\
& \alpha_{2 V}=\frac{1}{2 B_{4}} \sqrt{\frac{\left(B_{3}+A\right)^{2}}{4}-4 B_{4}\left(y+\frac{B_{3} y-B_{1}}{A}\right)}-\frac{B_{3}+A}{4 B_{4}},
\end{aligned}
$$

Here $A_{a b i j}=\frac{1}{\Theta L_{x} L_{y} L_{z}} \int_{0}^{\Theta}(\Theta-t) \iint_{0}^{L_{L}} \int_{0}^{L_{y}} \int_{0}^{L_{z}} k_{a, b}(x, y, z, T) I_{1}^{i}(x, y, z, t) V_{1}^{j}(x, y, z, t) d z d y d x d t, B_{4}=A_{I V 00}^{2} A_{I V 00}^{2}-$ $-2\left(A_{I V 00}^{2}-A_{I I 00} A_{V V 00}\right)^{2}, B_{3}=A_{I V 00} A_{I V 00}^{2}+A_{I V 01} A_{I V 00}^{3}+A_{I V 00} A_{I I 0} A_{I V 00}^{2}-4\left(A_{I V 00}^{2}-A_{I I 00} A_{V V 00}\right)\left[A_{I V 01} \times\right.$ $\left.\times 2 A_{I V 00}+2 A_{I V 00}\left(1+A_{I V 01}+A_{I I 10}\right)-2 A_{I I 00}\left(A_{I V 10}+A_{V V 10}+1\right)\right]-4 A_{I V 10} A_{I I 00} A_{I V 00}^{2}+2 A_{I V 00} A_{I V 01} A_{I V 00}^{2}$, $B_{2}=A_{I V 00}^{2}\left\{\left(1+A_{I V 01}+A_{I I 10}\right)^{2}+A_{I V 00}^{2} A_{I V 01}^{2}+2 A_{I V 00} A_{I V 00}\left(A_{I V 00}+A_{I V 00} A_{I V 01}+A_{I V 00} A_{I I 10}-4 A_{I V 10} A_{I I 00}\right)-\right.$ 


$$
\begin{aligned}
& \left.-4 A_{I I 00}\left[A_{I V 11}-A_{I I 20}-\frac{1}{L_{x} L_{y} L_{z}} \int_{0}^{L_{x} L_{y}} \int_{0}^{L_{z}} \int_{0}^{L_{I}} f_{I}(x, y, z) d z d y d x\right]\right\}\left\{\left[2 A_{I V 01} A_{I V 00}+2 A_{I V 00}\left(1+A_{I V 01}+A_{I I 10}\right)-\right.\right. \\
& \left.-2 A_{I I 00}\left(A_{I V 10}+1+A_{V V 10}\right)\right]^{2}+2\left[A_{I V 01}\left(1+A_{I V 01}+A_{I I 10}\right)+\frac{2}{L_{x} L_{y} L_{z}} \int_{0}^{L_{0}} \int_{0}^{L_{y} L_{z}} \int_{0} f_{V}(x, y, z) d z d y d x-2 A_{I I 00} \times\right. \\
& \left.\times\left(A_{V V 20}-A_{I V 11}\right)+A_{I V 01}\left(1+A_{I V 01}+A_{I I 10}\right)\right]\left[2 A_{I V 00}\left(1+A_{I V 01}+A_{I I 0}\right)+2 A_{I V 01} A_{I V 00}-2\left(A_{I V 10}+1+A_{V V 10}\right) \times\right. \\
& \left.\left.\times A_{I I 00}\right]\right\}, B_{1}=2 A_{I V 00} A_{I V 01}\left(1+A_{I V 01}+A_{I I 10}\right)^{2}-8\left[A_{I V 11}-A_{I I 20}-\frac{1}{L_{x} L_{y} L_{z}} \int_{0}^{L_{x}} \int_{0}^{L_{y}} \int_{0}^{L_{z}} f_{I}(x, y, z) d z d y d x\right]+ \\
& +A_{I V 00} A_{I V 01} A_{I I 00}+A_{I V 01}^{2}\left(A_{I V 00}+A_{I V 00} A_{I V 01}+A_{I V 00} A_{I I 10}-4 A_{I V 10} A_{I I 00}\right)-2\left[A_{I V 01}\left(1+A_{I V 01}+A_{I I 10}\right)+\right. \\
& \left.+\frac{2 A_{I I 00}}{L_{x} L_{y} L_{z}} \int_{0}^{L_{x} L_{y}} \int_{0}^{L_{z}} \int_{0} f_{I}(x, y, z) d z d y d x-2 A_{I I 00}\left(A_{V V 20}-A_{I V 11}\right)+A_{I V 01}\left(1+A_{I V 01}+A_{I I 10}\right)\right]\left[2 A _ { I V 0 0 } \left(1+A_{I V 01}+\right.\right. \\
& \left.\left.+A_{I I 10}\right)-2\left(A_{I V 10}+A_{V V 10}+1\right) A_{I I 00}+2 A_{I V 01} A_{I V 00}\right], B_{0}=4\left[\frac{1}{L_{x} L_{y} L_{z}} \int_{0}^{L_{x}} \int_{0}^{L_{y}} \int_{0}^{L_{z}} f_{I}(x, y, z) d z d y d x-A_{I V 11}+\right. \\
& \left.+A_{I I 20}\right] A_{I I 00} A_{I V 01}^{2}+A_{I V 01}^{2}\left(A_{I V 01}+A_{I I 10}+1\right)^{2}-\left[A_{I V 01}\left(1+A_{I V 01}+A_{I I 0}\right)-2 A_{I I 00}\left(A_{V V 20}-A_{I V 11}\right)+A_{I V 01} \times\right. \\
& \left.\times\left(1+A_{I V 01}+A_{I I 10}\right)+\frac{2 A_{I I 00}}{L_{x} L_{y} L_{z}} \int_{0}^{L_{x}} \int_{0}^{L_{y} L_{z}} \int_{0} f_{V}(x, y, z) d z d y d x\right]^{2}, y=\sqrt[3]{\sqrt{q^{2}+p^{3}}-q}-\sqrt[3]{\sqrt{q^{2}+p^{3}}+q}+\frac{B_{2}}{6}, \\
& q=\left(2 B_{1} B_{3}-8 B_{0}\right) \frac{B_{2}}{48}+\frac{B_{2}^{3}}{216}+\frac{B_{0}\left(4 B_{2}-B_{3}^{2}\right)-B_{1}^{2}}{8}, p=\frac{1}{72}\left[3\left(2 B_{1} B_{3}-8 B_{0}\right)-2 B_{2}^{2}\right], A=\sqrt{8 y+B_{3}^{2}-4 B_{2}}, \\
& \alpha_{2 \Phi_{I}}=A_{I I 20}-\frac{1}{\Theta L_{x} L_{y} L_{z}} \int_{0}^{\Theta}(\Theta-t) \int_{0}^{L_{x} L_{y} L_{z}} \int_{0}^{0} k_{I}(x, y, z, T) I(x, y, z, t) d z d y d x d t+ \\
& +\frac{1}{L_{x} L_{y} L_{z}} \int_{0}^{L_{x} L_{y} L_{z}} \int_{0} \int_{0} f_{\Phi I}(x, y, z) d z d y d x \\
& \alpha_{2 \Phi_{V}}=A_{V V 20}-\frac{1}{\Theta L_{x} L_{y} L_{z}} \int_{0}^{\Theta}(\Theta-t) \int_{0}^{L_{x} L_{y} L_{z}} \int_{0} k_{V}(x, y, z, T) V(x, y, z, t) d z d y d x d t+ \\
& +\frac{1}{L_{x} L_{y} L_{z}} \int_{0}^{L_{L}} \int_{0}^{L_{y} L_{z}} \int_{0} f_{\Phi V}(x, y, z) d z d y d x .
\end{aligned}
$$

After the substitution we obtain the equation for parameter $\alpha_{2 C}$ for any value of parameter $\gamma$. We analyzed distributions of concentrations of dopant and radiation defects in space and time by using the second-order approximations framework the method of averaged of function corrections. The obtained analytical results have been checked by comparison with results of numerical simulation.

\section{AuTHORS}

Pankratov Evgeny Leonidovich educated in Radiophysical department of Nizhny Novgorod State University (Doctor of Science). He has 190 published papers in area of his researches.

Bulaeva Elena Alexeevna was educated in Engineering and construction department of Nizhny Novgorod State University of Architecture and Civil Engineering (Master of Science). She has 130 published papers in area of her researches. 